\title{
External validation of current prediction systems of improvement after decompression surgery in Chiari malformation type I patients: can we do better?
}

\author{
James Feghali, MD, ${ }^{1}$ Yangyiran Xie, BS, ${ }^{2}$ Yuxi Chen, BS, ${ }^{2}$ Sean $L i,{ }^{3}$ and Judy Huang, MD' \\ 1Department of Neurosurgery, Johns Hopkins University School of Medicine; ${ }^{2}$ Krieger School of Arts and Sciences, Johns \\ Hopkins University, Baltimore, Maryland; and ${ }^{3}$ Pratt School of Engineering, Duke University, Durham, North Carolina
}

\begin{abstract}
OBJECTIVE The Chiari Severity Index (CSI) and points-based algorithm of Thakar et al. are two prognostic tools that have been developed to predict the likelihood of improvement after suboccipital decompression in adult patients with Chiari malformation type I (CM-I). This study aimed to externally validate and critically evaluate these algorithms in the interest of guiding the development of improved prediction systems.
\end{abstract}

METHODS A consecutive cohort of CM-I patients undergoing suboccipital decompression between September 2006 and September 2018 were included. The CSI and Thakar point score were computed for all patients, and associations with improvement were analyzed. The ability of both prediction systems to predict improvement as measured by different Chicago Chiari Outcome Scale (CCOS) cutoffs was assessed using receiver operating curve analysis. Post hoc correlations between the algorithms and different CCOS subcomponents were also assessed.

RESULTS The surgical cohort was composed of 149 adult CM-I patients, of whom $39(26 \%)$ had a syrinx. Most patients experienced improvement after surgery ( $80 \%$ CCOS $\geq 13 ; 96 \%$ CCOS $\geq 11)$. The proportion of patients improving decreased with increasing $\mathrm{CSI}$, but the results were not statistically significant $(p=0.246)$. No statistically significant difference in the mean Thakar point score was identified between improved and nonimproved patients using both CCOS cutoffs ( $p=0.246$ for a cutoff of 13 and $p=0.480$ for a cutoff of 11). The CSI had a poor ability in identifying improved patients at a CCOS cutoff of 13 (area under the curve [AUC] 0.582) and 11 (AUC 0.646). The Thakar point score similarly had poor discrimination at a cutoff of 13 (AUC 0.467) and 11 (AUC 0.646). Neither algorithm had significant correlation with any of the CCOS subcomponents except for CSI and nonpain symptom improvement (coefficient $=-0.273, p=$ $0.001)$.

CONCLUSIONS Previously published algorithms failed to provide prediction value with regard to clinically meaningful improvement following suboccipital decompression in adult CM-I patients. Future models and practical scoring systems are still required to improve the decision-making process.

https://thejns.org/doi/abs/10.3171/2020.2.JNS20181

KEYWORDS Arnold-Chiari malformation; decompression; surgical; syringomyelia; skull base

$\mathrm{C}$ HIARI malformation type I (CM-I), the most common Chiari malformation, has been classically described as caudal herniation of the cerebellar tonsils through the foramen magnum without involvement of the brainstem or fourth ventricle. ${ }^{1}$ CM-I remains poorly understood, and patients may report symptom persistence or reoccurrence after surgical decompression. ${ }^{2}$ The Chiari Severity Index (CSI) and the points-based algorithm by Thakar et al. are two prognostic tools designed to predict the likelihood of favorable outcome following suboccipital decompression in CM-I patients. ${ }^{3,4}$ The CSI was derived using a process of conjunctive consolidation from a cohort of pediatric CM-I patients and used both clinical and neuroimaging criteria to predict patient-defined outcome as reported in a 2 -item questionnaire. ${ }^{3}$ The point score of Thakar et al. was derived by conducting a logistic regression analysis on a cohort of 82 adult CM-I patients with syringomyelia and used clinical and neuroimaging variables to predict improvement based on the Chicago Chiari Outcome Scale (CCOS). ${ }^{4}$ The ability of these tools to reliably predict clinically significant improvement after decompression surgery in adult CM-I patients remains unclear.

ABBREVIATIONS AUC $=$ area under the curve CCOS $=$ Chicago Chiari Outcome Scale; $\mathrm{CM}-\mathrm{I}=$ Chiari malformation type I; CSI = Chiari Severity Index; $\mathrm{ROC}=$ receiver operating characteristic.

SUBMITTED January 17, 2020. ACCEPTED February 18, 2020.

INCLUDE WHEN CITING Published online May 1, 2020; DOI: 10.3171/2020.2.JNS20181. 


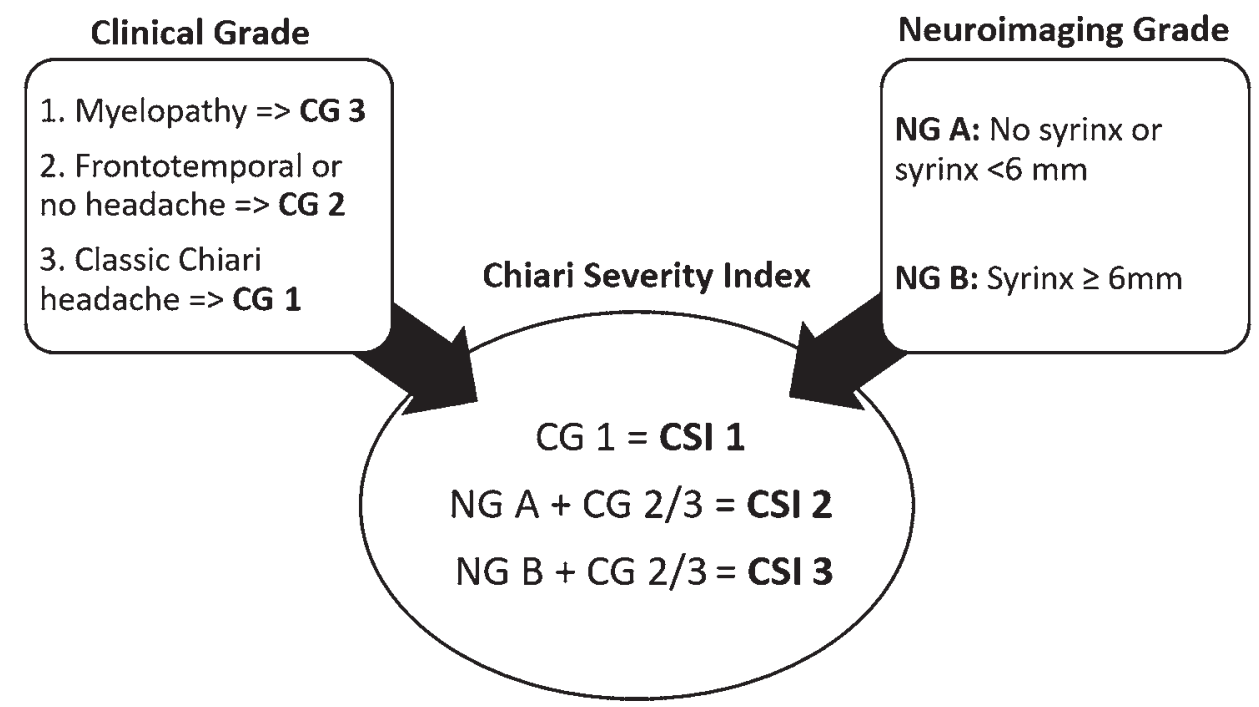

FIG. 1. Criteria used to derive the clinical grade (CG), neuroimaging grade (NG), and final CSI.

In the absence of evidence-based guidelines for operative management of adult symptomatic CM-I, patient selection for surgical intervention constitutes a difficult and complex decision-making process, especially given the nonspecific nature of symptoms and overlap with other diagnoses. This study aimed to externally validate and critically evaluate current scoring systems to identify areas for potential improvement in outcome prediction.

\section{Methods}

\section{Patient Population}

Prospectively accrued data on adult (age $>18$ years) CM-I patients undergoing suboccipital decompression at Johns Hopkins Medical Institutions between September 1, 2006, and September 1, 2018, were housed in an institutional review board-approved database used for this study. Since this was a retrospective analysis that did not involve patient contact, consent from patients was not needed. All CM-I patients were symptomatic and had at least $3 \mathrm{~mm}$ of tonsillar tip herniation beyond the McRae line on sagittal MRI. The cohort consisted of patients with and without a spinal cord syrinx. A syrinx was defined as at least 3 $\mathrm{mm}$ of cavitation in the spinal cord on T2-weighted MRI. ${ }^{3}$ Exclusion criteria included reoperation procedures and patients with concomitant connective tissue disease (e.g., Ehlers-Danlos syndrome).

\section{Surgical Procedure}

A thorough evaluation of clinical presentation, symptom severity, limitations in daily activities, imaging, and CSF flow studies was conducted before offering surgery to patients. Indications for surgery generally included clinically impairing symptoms that affected daily life or any degree of symptomatic syringomyelia. The senior author (J.H.) performed all surgeries, which consisted of suboccipital craniectomy, C1 laminectomy, duraplasty, and cranioplasty. Durepair (Medtronic), a nonsynthetic dura substitute, was used for duraplasty, and the cranioplasty was performed using the Medpor Titan Barrier implant (Stryker), a titanium mesh embedded in polyethylene. The implant is cut to size and fixed over the craniectomy using 5-mm screws. The intention of the cranioplasty was to minimize the incidence of cerebellar slumping postoperatively and to prevent adhesions caused by cervical muscles. Arachnoid dissection was carried out in patients with a syrinx, and tonsillopexy with bipolar cautery was performed for tonsils with more than $10 \mathrm{~mm}$ of herniation beyond the foramen magnum (18 cases).

\section{Baseline Prediction Variables}

Demographic, clinical, and imaging characteristics were extracted from patient records. Particular attention was paid toward extracting clinical variables constituting the CSI: the presence, location, and quality of headaches as well as myelopathic symptoms such as numbness, weakness, hyperreflexia, and unsteady gait. The CSI neuroimaging variables of syrinx presence and maximum cross-sectional diameter were also recorded. Variables constituting the points-based algorithm of Thakar et al. were also extracted and included obex position (OP) in millimeters and $\mathrm{M}$-line-fourth ventricle vertex distance (M-line-FVV) in millimeters as measured in the original paper. ${ }^{4}$ Subsequently, the CSI (Fig. 1) and composite Thakar point score $($ score $=162-[10 \times$ gait instability + OP + M-line-FVV]) were calculated for every patient. Since numbness is a subjective symptom that is commonly reported in CM-I, patients were only considered as being myelopathic when numbness was accompanied by other myelopathic symptoms such as weakness.

\section{Outcome Assessment and Statistical Analysis}

The CCOS, a validated tool consisting of 4 components, was used for validation. ${ }^{5}$ To calculate the final CCOS score, the extent of improvement in pain symptoms, nonpain symptoms, and functional status were recorded 
TABLE 1. Baseline characteristics of adult CM-I patients with and without improvement after decompression surgery

\begin{tabular}{|c|c|c|c|}
\hline Characteristic & $\begin{array}{l}\text { Improvement } \\
(\mathrm{CCOS} \geq 13)\end{array}$ & $\begin{array}{l}\text { No Improvement } \\
\quad(\text { CCOS <13) }\end{array}$ & $\begin{array}{c}p \\
\text { Value }\end{array}$ \\
\hline No. of patients & 119 & 30 & \\
\hline Age at diagnosis, yrs & $36.8 \pm 12.3$ & $39.6 \pm 9.5$ & 0.265 \\
\hline Sex & & & 0.765 \\
\hline Female & $98(82)$ & $24(80)$ & \\
\hline Male & $21(18)$ & $6(20)$ & \\
\hline Race & & & $0.044^{*}$ \\
\hline White & $77(65)$ & $25(83)$ & \\
\hline Black & $32(27)$ & $2(7)$ & \\
\hline Other & $10(8)$ & $3(10)$ & \\
\hline $\begin{array}{l}\text { Duration of symptoms } \\
\text { before surgery, yrs }\end{array}$ & $5.6 \pm 6.4$ & $4.1 \pm 4.9$ & 0.233 \\
\hline \multicolumn{4}{|l|}{ Clinical presentation } \\
\hline Headache & $113(95)$ & $28(93)$ & 0.662 \\
\hline Valsalva headache & $78(66)$ & $13(43)$ & $0.026^{*}$ \\
\hline Occipital headache $†$ & $67(59)$ & $16(57)$ & 0.836 \\
\hline Numbness & $70(59)$ & $16(53)$ & 0.586 \\
\hline Imbalance & $78(66)$ & $19(63)$ & 0.820 \\
\hline Weakness & $22(19)$ & $12(40)$ & $0.012^{*}$ \\
\hline Hyperreflexia & $5(71)$ & $2(29)$ & 0.629 \\
\hline \multicolumn{4}{|l|}{ Radiological variables } \\
\hline Syrinx present & $28(24)$ & $11(37)$ & 0.144 \\
\hline Obex position, mm & $8.9 \pm 3.5$ & $8.6 \pm 3.0$ & 0.733 \\
\hline M-line-FVV distance, $\mathrm{mm}$ & $30.8 \pm 3.4$ & $30.2 \pm 3.4$ & 0.378 \\
\hline
\end{tabular}

at last follow-up, and postoperative complications were noted. Associations between the CSI and the point score of Thakar et al. were tested using the ANOVA test with a post hoc comparison using Bonferroni adjustment. Although the CSI paper used a simplified and nonvalidated patient survey for outcome assessment, ${ }^{3}$ Thakar et al. defined favorable outcome as a composite CCOS score of 11 or more. ${ }^{4}$ In addition to this cutoff, an analysis of improvement defined by a score of 13 or more (as suggested by the original CCOS paper $)^{5}$ was also conducted. The Fisher exact test was used to compare improvement across different CSIs, and the independent-samples t-test was used to compare point scores from the Thakar algorithm between improved and nonimproved patients. For CSI, a logistic regression model was created with the CSI grade entered as a categorical predictor and CCOS-based improvement as the outcome. Predicted probabilities of this model and the total points yielded from the formula of Thakar et al. were assessed using receiver operating characteristic (ROC) curve analysis to evaluate the ability to discriminate between improvement and no improvement. A post hoc analysis evaluating correlation coefficients between the CSI and Thakar point score on one hand and differ-
TABLE 2. Number of patients in each CSI with breakdown by clinical and neuroimaging grade

\begin{tabular}{|c|c|c|c|c|}
\hline \multirow{2}{*}{$\begin{array}{c}\text { Neuroimaging } \\
\text { Grade }\end{array}$} & \multicolumn{3}{|c|}{ Clinical Grade } & \multirow[b]{2}{*}{ Total } \\
\hline & 1 & 2 & 3 & \\
\hline A & CSI 1: 69 pts & CSI 2: 16 pts & CSI 2: 45 pts & $130 \mathrm{pts}$ \\
\hline$B$ & CSI 1: 9 pts & CSI 3: 4 pts & CSI 3: 6 pts & 19 pts \\
\hline Total & $78 \mathrm{pts}$ & 20 pts & $51 \mathrm{pts}$ & $149 \mathrm{pts}$ \\
\hline
\end{tabular}

Pts = patients.

ent CCOS subcomponent scores on the other was also performed using Pearson correlation. Statistical analyses were performed using IBM SPSS software (version 25.0, IBM Corp.) with statistical significance set at $\mathrm{p}<0.05$.

\section{Results}

\section{Patients and Baseline Characteristics}

Of 220 adult CM-I patients presenting for care, surgery was performed in $149 \mathrm{CM}-\mathrm{I}$ patients (mean age 35.1 years, SD 11.5 years). Demographically, most patients were white $(102 / 149,69 \%)$ and female $(122 / 149,82 \%)$. Patients had an average duration of symptoms of 5.3 years (SD 6.1 years) before surgery. The most common symptom reported was headache $(141 / 149,95 \%)$, and most headaches were exacerbated by Valsalva maneuver $(91 / 141,65 \%)$. Most patients $(139 / 149,93 \%)$ had tonsillar ectopia of $5 \mathrm{~mm}$ or more. The distribution of tonsillar ectopia in the 149 patients was as follows: 10 (7\%) from 3.0 to $4.9 \mathrm{~mm}, 97$ (65\%) from 5.0 to $10.0 \mathrm{~mm}$, and $42(28 \%)$ beyond $10.0 \mathrm{~mm}$. The prevalence of spinal cord syrinx was $26 \%$ (39/149), and 19 of these patients had a maximum diameter greater than $8 \mathrm{~mm}$. Baseline characteristics compared by dichotomous CCOS improvement are provided in Table 1.

\section{Preoperative Improvement Scores}

Most patients $(78 / 149,52 \%)$ had a CSI of 1 , while few $(10 / 149,7 \%)$ had a CSI of 3 (Table 2 ). The obex position or M-line-FVV distance was missing for 6 patients $(<5 \%)$, so the Thakar point score could be calculated for $143 \mathrm{pa}-$ tients. According to the formula of Thakar et al., the mean point score for the entire cohort was 116.0 (SD 6.8). As represented by Fig. 2, there was no significant difference in the mean point score among different CSIs (overall ANOVA, $\mathrm{p}=0.197$ ).

\section{Complications}

Procedure-related complications occurred in $16 \%$ (24/149) of the CM-I patients. The breakdown of complications was as follows: surgical site infection (12/24, $50 \%$ ), chemical meningitis $(5 / 24,21 \%)$, and pseudomeningocele $(7 / 24,29 \%)$. The majority of the complications were transient $(14 / 24,58 \%)$ with no significant impact on long-term outcome. Operative reexploration was required in 4 patients: 1 developed a 7-cm pseudomeningocele, 2 required removal of infected cranioplasties, and 1 underwent expansion of craniectomy due to symptom return on follow-up. 


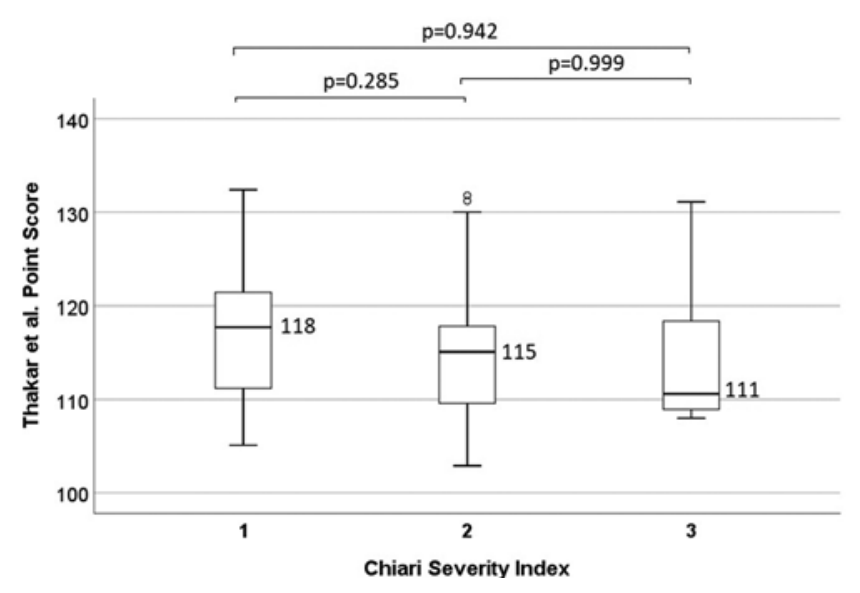

FIG. 2. Boxplots of the Thakar point score across different CSIs.

\section{Outcome and Improvement Prediction}

The mean CCOS score for the cohort was 13.8 (SD $1.7)$, and most patients $(119 / 149,80 \%)$ experienced improvement $(\mathrm{CCOS} \geq 13)$ after 1.9 years of postoperative follow-up. Using a CCOS cutoff of 11 or more, 143 (96\%) patients experienced improvement. The proportion of patients with improvement $(\mathrm{CCOS} \geq 13)$ decreased with increasing CSIs: $85 \%$ improvement for CSI 1, 75\% improvement for CSI 2, and 70\% improvement for CSI 3, but the results were not statistically significant $(\mathrm{p}=0.246)$. Using a CCOS cutoff of 13 or more, there was no statistically significant difference in the mean point score of improved (115.8, SD 6.6) and nonimproved (116.8, SD 7.7) patients ( $\mathrm{p}$ $=0.480$ ). When a cutoff of 11 or more was used, improved patients had a higher mean point score than nonimproved patients (116.1 vs 112.7, $\mathrm{p}=0.236$ ), but the results were not statistically significant. On ROC analysis, the CSI had a poor ability of discriminating between improved and nonimproved patients with both CCOS cutoffs of 13 (area under the curve [AUC] 0.582, 95\% CI 0.467-0.697; $\mathrm{p}=0.166$ ) and 11 (AUC 0.646, 95\% CI 0.436-0.856; $\mathrm{p}$ $=0.227$ ). The Thakar point score similarly demonstrated poor discrimination with a cutoff of 13 (AUC $0.467,95 \%$ CI $0.342-0.592 ; \mathrm{p}=0.584$ ) and 11 (AUC $0.646,95 \% \mathrm{CI}$ $0.454-0.838 ; \mathrm{p}=0.227$ ). The ROC analysis is summarized in Fig. 3. Pearson correlation showed no significant correlation between the CSI and any of the CCOS subcomponents except nonpain symptom improvement (correlation coefficient $=-0.273, \mathrm{p}=0.001$ ). Similarly, the Thakar point score showed no significant correlation with any of the CCOS subcomponents.

\section{Discussion}

There is considerable controversy revolving around the management of CM-I, which mostly stems from incomplete understanding of disease pathophysiology and natural history. ${ }^{6}$ Procedure-related complications can increase morbidity, ${ }^{7}$ while some untreated patients may experience a benign natural history. ${ }^{8}$ Hence, the proper selection of adult CM-I patients for surgery is crucial.

\section{The Chiari Severity Index}

In our surgical cohort of adult CM-I patients, the CSI was very limited in its ability to predict improved outcome using both cutoffs of CCOS. There are several possible reasons that explain this finding. The original cohort from which the CSI was derived consisted solely of pediatric patients, and the authors mentioned in the limitations that "external validation in other centers and in adult populations is necessary." 3 Grangeon et al. tested the CSI on 38 adult CM-I patients; the proportion of improved patients $(\mathrm{CCOS} \geq 13)$ decreased with increasing CSI, but results were not statistically significant, similar to the results of our study. ${ }^{9}$ This weak association between CSI and outcomes in the adult population may be the result of important differences between adult and pediatric CM-I. A study by Gilmer at al. showed that pediatric patients $(\leq 18$ years old) respond better to decompression when compared with young and old adults. ${ }^{10}$ Dlouhy et al. reported differences in the intradural pathology identified intraoperatively when adult and pediatric CM-I patients were compared. ${ }^{11}$ These and other differences between pediatric and adult CM-I patients partly underlie the variation in operative management of these two patient groups. Namely, a comprehensive literature review showed that the dura was opened and the arachnoid was dissected much less commonly in pediatric surgical series, in an attempt to limit CSF-related complications. ${ }^{12}$ Tonsillar resection, however, was around twice as common in pediatric series compared with adult cohorts. ${ }^{12}$ The details (duraplasty, $\mathrm{C} 1$ laminectomy, cranioplasty, tonsillopexy) and homogeneity of the surgical procedures were not reported in the CSI study and may differ from the uniform decompression surgery performed in our cohort. In addition, around $20 \%$ of the pediatric patients in the CSI study were younger than 5 years $;^{13}$ consequently, inaccurate articulation of symptoms by patients and their parents may have compromised the validity and reliability of the index.

Another important limitation of the CSI study was the use of a nonvalidated quality-of-life assessment consisting of 2 brief questions to detect improvement. The authors mentioned that "the performance of the CSI should be tested against novel metrics." ${ }^{3}$ We selected the CCOS as an outcome tool since its validity and reliability have been verified previously. ${ }^{14,15}$ Once the CSI was tested using this standardized metric, it demonstrated limited discrimination between improved and nonimproved patients.

\section{The Points-Based Algorithm of Thakar et al.}

The point score of Thakar et al. demonstrated poor discrimination regardless of the CCOS cutoff used. The point score itself was developed from a cohort of adult CM-I patients who all had syringomyelia, and the CCOS cutoff used for improvement in the original paper was 11 or more. ${ }^{4}$ The score appears to have limited generalizability when applied to a mixed cohort of adult CM-I patients with and without syringomyelia. Moreover, in any bedside predictive tool, the chosen outcome and its definition should always have clinical relevance. This has been emphasized by Parker et al., who stressed on the importance of detecting critical improvement thresholds that are clinically meaningful for CM-I patients. ${ }^{16}$ A CCOS cutoff 

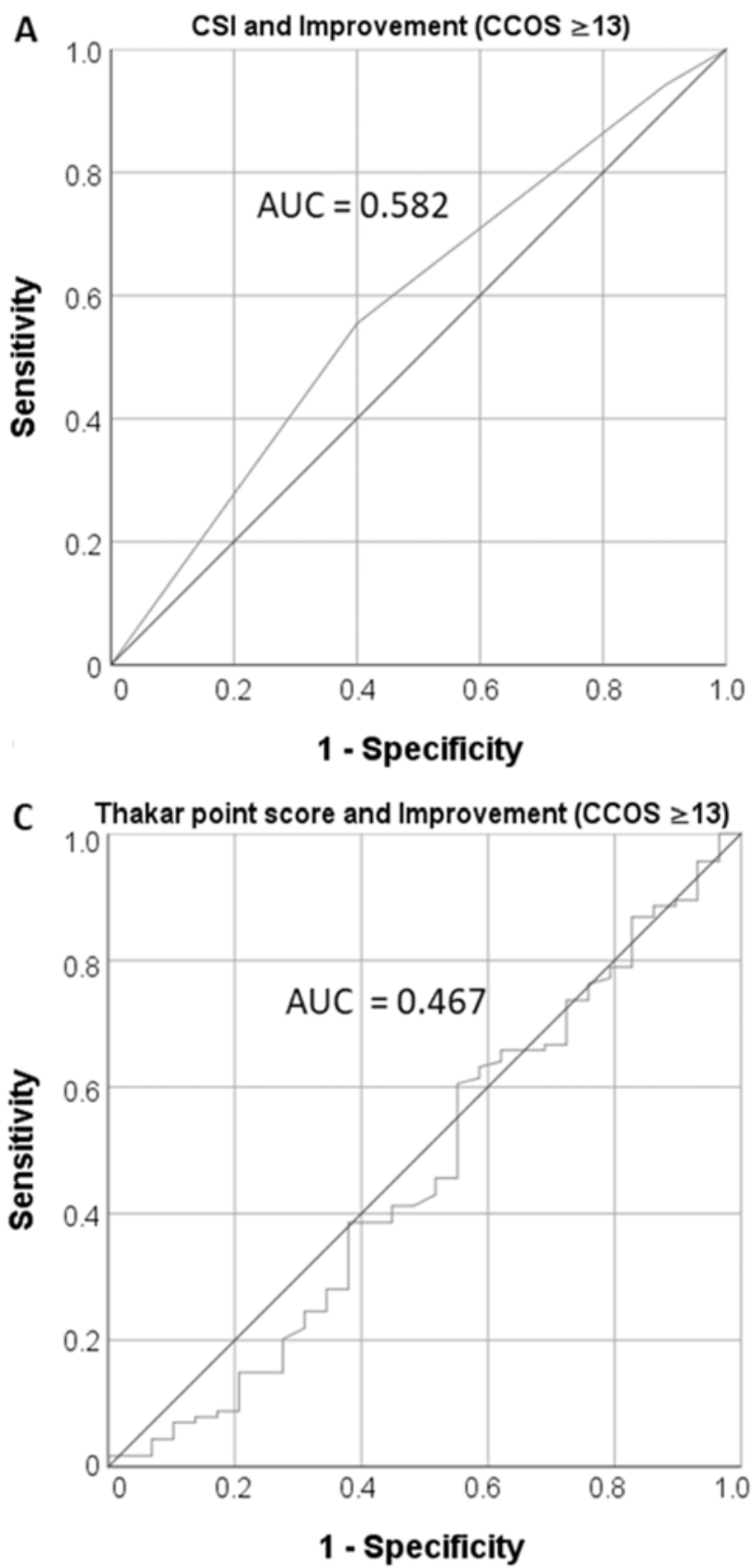
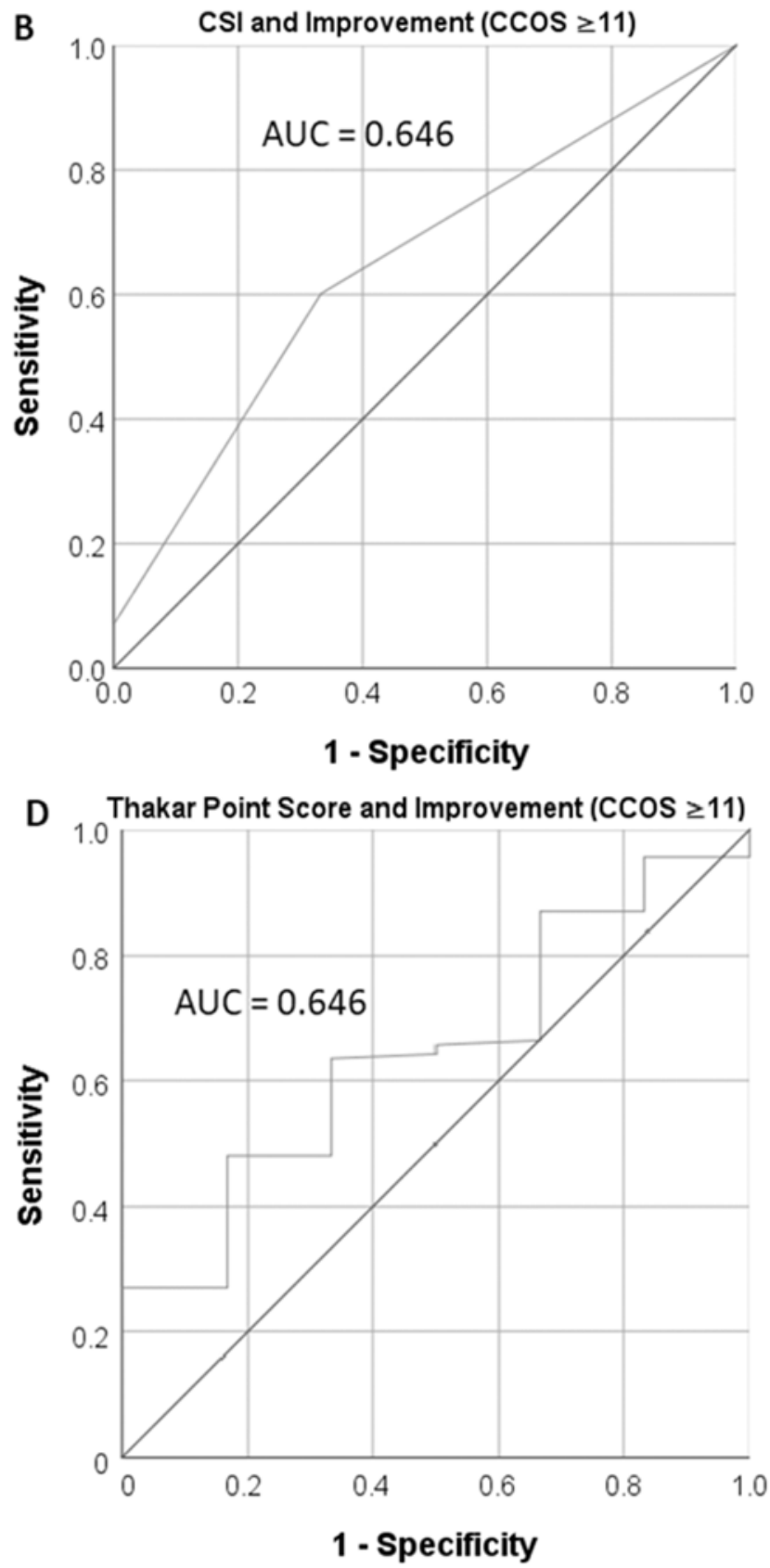

FIG. 3. Ability of the CSI (A and B) and Thakar point score (C and D) to discriminate between improvement and no improvement based on the CCOS.

of 11 or more includes scores of 11 and 12 , which, according to the original CCOS paper, correlate best with an "unchanged" gestalt outcome. This limits the utility of the score in real-world clinical practice. Moreover, external validation of the CCOS has indicated that even more conservative cutoffs such as 14 or more may correlate better with improved outcome after decompression. ${ }^{17}$ When the score was tested using a more conservative cutoff in our cohort, the performance deteriorated considerably (AUC 0.467). Furthermore, another essential attribute of clini- cally useful prediction systems is practicality and usability. Although the authors rigorously assessed several putative clinical and imaging variables, the final formula and its interpretation are somewhat tedious and are expected to lead to poor adoption rates.

\section{Future Models}

The previously published models of improvement prediction have laid a foundation for designing better prognostic systems for adult CM-I patients. Such an endeavor 
should start by selecting validated outcome tools with clinically meaningful cutoffs (e.g., CCOS $\geq 14$ ). The statistical methods used should also ensure the absence of significant overfitting by utilizing a holdout method with a validation data set or bootstrapping techniques. Additionally, the cohort should be composed of an adequate sample size and of a heterogeneous population of CM-I patients with and without syringomyelia that represent the adult CM-I population typically seen in clinic. Ideally, there should be a comprehensive assessment of many previously described variables, including clinical and imaging characteristics. The details of the suboccipital decompression procedure should also be provided and preferably standardized to avoid distorting associations between baseline characteristics and outcome. To maximize the likelihood of adoption, the scoring system design should be parsimonious and user-friendly. Providing a simple web-based platform to test the prediction tool can also promote its use and validation.

\section{Limitations}

The results of our analysis must be interpreted with caution. We retrospectively evaluated the performance of previously published models on a single-center cohort of adult CM-I patients. This specific cohort may not be representative of the adult CM-I population at large, and therefore, additional validation efforts, preferably prospective, are encouraged. Given the lack of guidelines, there is considerable variation in the exact surgical methods used in decompression. In the absence of complicating factors, the surgeon in this study consistently performs duraplasty and cranioplasty when treating adult CM-I patients. As this may affect the performance of the previously published models, validation in cohorts using other surgical techniques is also advised.

\section{Conclusions}

We have evaluated the performance of published improvement prediction models while critically evaluating their clinical usefulness. In general, our external validation indicated suboptimal performance and limited utility of these models, and our analysis yielded several areas of improvement that can guide the development of future models.

\section{References}

1. Milhorat TH, Chou MW, Trinidad EM, et al. Chiari I malformation redefined: clinical and radiographic findings for 364 symptomatic patients. Neurosurgery. 1999;44(5):1005-1017.

2. Sivaramakrishnan A, Alperin N, Surapaneni S, Lichtor T. Evaluating the effect of decompression surgery on cerebrospinal fluid flow and intracranial compliance in patients with Chiari malformation with magnetic resonance imaging flow studies. Neurosurgery. 2004;55(6):1344-1351.

3. Greenberg JK, Yarbrough CK, Radmanesh A, et al. The Chiari Severity Index: a preoperative grading system for Chiari malformation type 1. Neurosurgery. 2015;76(3):279-285.

4. Thakar S, Sivaraju L, Jacob KS, et al. A points-based algorithm for prognosticating clinical outcome of Chiari malformation Type I with syringomyelia: results from a predictive model analysis of 82 surgically managed adult patients. $J$ Neurosurg Spine. 2018;28(1):23-32.
5. Aliaga L, Hekman KE, Yassari R, et al. A novel scoring system for assessing Chiari malformation type I treatment outcomes. Neurosurgery. 2012;70(3):656-664.

6. Luciano MG, Batzdorf U, Kula RW, et al. Development of Common Data Elements for use in Chiari malformation type I clinical research: an NIH/NINDS project. Neurosurgery. 2019;85(6):854-860.

7. Greenberg JK, Ladner TR, Olsen MA, et al. Complications and resource use associated with surgery for Chiari malformation type 1 in adults: a population perspective. Neurosurgery. 2015;77(2):261-268.

8. Langridge B, Phillips E, Choi D. Chiari malformation type 1: a systematic review of natural history and conservative management. World Neurosurg. 2017;104:213-219.

9. Grangeon L, Puy L, Gilard V, et al. Predictive factors of headache resolution after Chiari type 1 malformation surgery. World Neurosurg. 2018;110:e60-e66.

10. Gilmer HS, Xi M, Young SH. Surgical decompression for Chiari malformation type I: an age-based outcomes study based on the Chicago Chiari Outcome Scale. World Neurosurg. 2017; 107:285-290.

11. Dlouhy BJ, Dawson JD, Menezes AH. Intradural pathology and pathophysiology associated with Chiari I malformation in children and adults with and without syringomyelia. $J$ Neurosurg Pediatr. 2017;20(6):526-541.

12. Arnautovic A, Splavski B, Boop FA, Arnautovic KI. Pediatric and adult Chiari malformation Type I surgical series 1965-2013: a review of demographics, operative treatment, and outcomes. J Neurosurg Pediatr. 2015;15(2):161-177.

13. Greenberg JK, Yarbrough CK, Radmanesh A, et al. In reply: Chiari Severity Index: a novel grading system intended for preoperative counseling. Neurosurgery. 2015;77(5):E842.

14. Greenberg JK, Milner E, Yarbrough CK, et al. Outcome methods used in clinical studies of Chiari malformation Type I: a systematic review. J Neurosurg. 2015;122(2):262-272.

15. Yarbrough CK, Greenberg JK, Park TS. Clinical outcome measures in Chiari I malformation. Neurosurg Clin N Am. 2015;26(4):533-541.

16. Parker SL, Godil SS, Zuckerman SL, et al. Comprehensive assessment of 1-year outcomes and determination of minimum clinically important difference in pain, disability, and quality of life after suboccipital decompression for Chiari malformation I in adults. Neurosurgery. 2013;73(4):569-581.

17. Yarbrough CK, Greenberg JK, Smyth MD, et al. External validation of the Chicago Chiari Outcome Scale. J Neurosurg Pediatr. 2014;13(6):679-684.

\section{Disclosures}

Dr. Huang: stock ownership interest in Longeviti Neuro Solutions.

\section{Author Contributions}

Conception and design: all authors. Acquisition of data: Feghali, Xie, Chen, Li. Analysis and interpretation of data: Feghali. Drafting the article: all authors. Critically revising the article: Huang, Feghali, Xie, Chen. Reviewed submitted version of manuscript: all authors. Approved the final version of the manuscript on behalf of all authors: Huang. Statistical analysis: Feghali. Study supervision: Huang.

\section{Correspondence}

Judy Huang: Johns Hopkins Hospital, Baltimore, MD. jhuang24@ jhmi.edu. 